\title{
Critical Sets in Bipartite Graphs
}

\author{
Vadim E. Levit \\ Department of computer Science and Mathematics \\ Ariel University Center of Samaria, Israel \\ levitv@ariel.ac.il \\ Eugen Mandrescu \\ Department of computer Science \\ Holon Institute of Technology, Israel \\ eugen_m@hit.ac.il
}

\begin{abstract}
Let $G=(V, E)$ be a graph. A set $S \subseteq V$ is independent if no two vertices from $S$ are adjacent, and by $\operatorname{Ind}(G)(\Omega(G))$ we mean the set of all (maximum) independent sets of $G$, while $\alpha(G)=|S|$ for $S \in \Omega(G)$, and core $(G)=\cap\{S: S \in \Omega(G)\}$ [6]. The neighborhood of $A \subseteq V$ is denoted by $N(A)=\{v \in V: N(v) \cap A \neq \emptyset\}$, where $N(v)$ is the neighborhood of the vertex $v$. The number $d(X)=|X|-|N(X)|$ is the difference of the set $X \subseteq V$, and $d_{c}(G)=\max \{d(I): I \in \operatorname{Ind}(G)\}$ is called the critical difference of $G$. A set $X$ is critical if $d(X)=d_{c}(G)$ [14.

For a graph $G$ we define $\operatorname{ker}(G)=\cap\{S: S$ is a critical independent set $\}$, while $\operatorname{diadem}(G)=\cup\{S: S$ is a critical independent set $\}$.

For a bipartite graph $G=(A, B, E)$, with bipartition $\{A, B\}$, Ore 11 defined $\delta(X)=d(X)$ for every $X \subseteq A$, while $\delta_{0}(A)=\max \{\delta(X): X \subseteq A\}$. Similarly is defined $\delta_{0}(B)$.

In this paper we prove that for every bipartite graph $G=(A, B, E)$ the following assertions hold:

- $d_{c}(G)=\delta_{0}(A)+\delta_{0}(B)$;

- $\operatorname{ker}(G)=\operatorname{core}(G)$

- $|\operatorname{ker}(G)|+|\operatorname{diadem}(G)|=2 \alpha(G)$.
\end{abstract}

Keywords: maximum independent set, maximum matching, critical set, critical difference, König-Egerváry graph.

\section{Introduction}

Throughout this paper $G=(V, E)$ is a simple (i.e., a finite, undirected, loopless and without multiple edges) graph with vertex set $V=V(G)$ and edge set $E=E(G)$. If $X \subseteq V$, then $G[X]$ is the subgraph of $G$ spanned by $X$. By $G-W$ we mean either the subgraph $G[V-W]$, if $W \subseteq V(G)$, or the partial subgraph $H=(V, E-W)$ of $G$, for 
$W \subseteq E(G)$. In either case, we use $G-w$, whenever $W=\{w\}$. By $G=(A, B, E)$ we denote a bipartite graph having $\{A, B\}$ as a bipartition and we assume that $A \neq \emptyset \neq B$.

The neighborhood of a vertex $v \in V$ is the set $N(v)=\{w: w \in V$ and $v w \in E\}$, while the neighborhood of $A \subseteq V$ is denoted by $N(A)=N_{G}(A)=\{v \in V: N(v) \cap A \neq \emptyset\}$, and $N[A]=N(A) \cup A$.

A matching is a set of non-incident edges of $G$; a matching of maximum cardinality $\mu(G)$ is a maximum matching, and a perfect matching is a matching covering all the vertices of $G$. If $M$ is a matching, then $M(v)$ means the mate of the vertex $v$ by $M$, and $M(X)=\{M(v): v \in X\}$ for $X \subset V(G)$.

A set $S \subseteq V(G)$ is independent (or stable) if no two vertices from $S$ are adjacent, and by $\operatorname{Ind}(G)$ we denote the set of all independent sets of $G$. An independent set of maximum size will be referred to as a maximum independent set of $G$, and the independence number of $G$ is $\alpha(G)=\max \{|S|: S \in \operatorname{Ind}(G)\}$. Let $\Omega(G)$ be the family of all maximum independent sets of $G$, and $\operatorname{core}(G)=\cap\{S: S \in \Omega(G)\}[6$.

Recall from 14 the following definitions for a graph $G=(V, E)$ :

- $d(X)=|X|-|N(X)|, X \subseteq V$ is the difference of the set $X$;

- $d_{c}(G)=\max \{d(X): X \subseteq V\}$ is the critical difference of $G$;

- a set $U \subseteq V$ is $d$-critical if $d(U)=d_{c}(G)$;

- $i d_{c}(G)=\max \{d(I): I \in \operatorname{Ind}(G)\}$ is the critical independence difference of $G$;

- if $A \subseteq V$ is independent and $d(A)=i d_{c}(G)$, then $A$ is critical independent.

For a graph $G$ let us denote

$$
\begin{aligned}
\operatorname{ker}(G) & =\cap\{S: S \subseteq V \text { is a critical independent set }\}, \\
\text { diadem }(G) & =\cup\{S: S \subseteq V \text { is a critical independent set }\} .
\end{aligned}
$$

For instance, the graph $G_{1}$ from Figure 1 has $X=\{x, y, z, u, v\}$ as a critical set, because $N(X)=\{a, b, u, v\}$ and $d(X)=1=d_{c}\left(G_{1}\right)$. In addition, let us notice that $\operatorname{ker}\left(G_{1}\right)=\{x, y\} \subset \operatorname{core}\left(G_{1}\right)$, and $\operatorname{diadem}\left(G_{1}\right)=\{x, y, z\}$. The graph $G_{2}$ from Figure 1 has $d_{c}\left(G_{1}\right)=d\left(\left\{v_{1}, v_{2}\right\}\right)=\left|\left\{v_{1}, v_{2}\right\}\right|-\left|N\left(\left\{v_{1}, v_{2}\right\}\right)\right|=1$. It is easy to see that $\operatorname{core}\left(G_{1}\right)$ is a critical set, while $\operatorname{core}\left(G_{2}\right)$ is not a critical set, but $\operatorname{ker}\left(G_{2}\right)=\left\{v_{1}, v_{2}\right\} \subset \operatorname{core}\left(G_{2}\right)$.
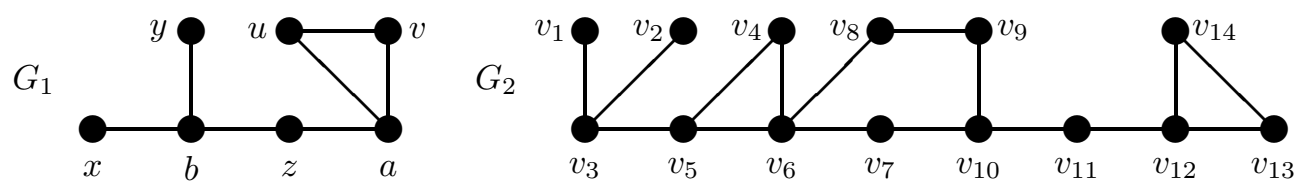

Figure 1: $\operatorname{core}\left(G_{1}\right)=\{x, y, z\}$, while $\operatorname{core}\left(G_{2}\right)=\left\{v_{1}, v_{2}, v_{7}, v_{11}\right\}$.

The following results will be used in the sequel. 
Theorem 1.1 Let $G$ be a graph. Then the following assertions are true:

(i) 14 d $d_{c}(G)=i d_{c}(G)$.

(ii) [5] There is a matching from $N(S)$ into $S$, for every critical independent set $S$.

(iii) [1] Each critical independent set is contained in a maximum independent set.

(iv) [10] the function d is supermodular, i.e.,

$$
d(X \cup Y)+d(X \cap Y) \geq d(X)+d(Y) \text { for every } X, Y \subseteq V(G) ;
$$

(v) [10] if $S_{1}, S_{2}$ are d-critical sets, then $S_{1} \cap S_{2}, S_{1} \cup S_{2}$ are d-critical as well;

(vi) [10] there is a unique minimal d-critical set, namely, $\operatorname{ker}(G)$.

(vii) [10] $\operatorname{ker}(G) \subseteq \operatorname{core}(G)$.

If $\alpha(G)+\mu(G)=|V(G)|$, then $G$ is called a König-Egerváry graph, 2, [13. It is well-known that each bipartite graph enjoys this property [3], 4$]$.

Theorem 1.2 If $G=(V, E)$ is a König-Egerváry graph, $M$ is a maximum matching, and $S \in \Omega(G)$, then:

(i) [7] $M$ matches $V-S$ into $S$, and $N(\operatorname{core}(G))$ into $\operatorname{core}(G)$;

(ii) [8] $S$ is d-critical, and $d_{c}(G)=\alpha(G)-\mu(G)=|\operatorname{core}(G)|-|N(\operatorname{core}(G))|$.

Following Ore [11, 12, the number

$$
\delta(X)=d(X)=|X|-|N(X)|
$$

is called the deficiency of $X$, where $X \subseteq A$ or $X \subseteq B$ and $G=(A, B, E)$ is a bipartite graph. Let

$$
\delta_{0}(A)=\max \{\delta(X): X \subseteq A\}, \quad \delta_{0}(B)=\max \{\delta(Y): Y \subseteq B\} .
$$

A subset $X \subseteq A$ having $\delta(X)=\delta_{0}(A)$ is called $A$-critical, while $Y \subseteq B$ having $\delta(B)=\delta_{0}(B)$ is called $B$-critical. For a bipartite graph $G=(A, B, E)$ let us denote $\operatorname{ker}_{A}(G)=\cap\{S: S$ is $A$-critical $\}$ and $\operatorname{diadem}_{A}(G)=\cup\{S: S$ is $A$-critical $\}$. Similarly, $\operatorname{ker}_{B}(G)=\cap\{S: S$ is $B$-critical $\}$ and $\operatorname{diadem}_{B}(G)=\cup\{S: S$ is $B$-critical $\}$.

It is convenient to define $d(\emptyset)=\delta(\emptyset)=0$.

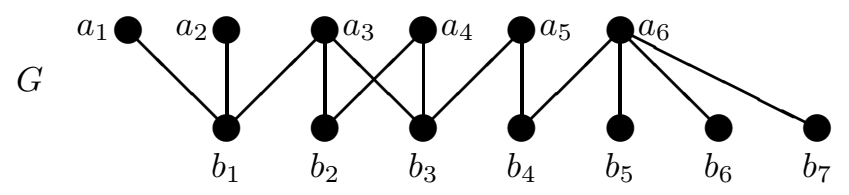

Figure 2: $G$ is a bipartite graph without perfect matchings.

For instance, the graph $G=(A, B, E)$ from Figure 2 has: $X=\left\{a_{1}, a_{2}, a_{3}, a_{4}\right\}$ as an $A$-critical set, $\operatorname{ker}_{A}(G)=\left\{a_{1}, a_{2}\right\}, \operatorname{diadem}_{A}(G)=\left\{a_{i}: i=1, \ldots, 5\right\}$ and $\delta_{0}(A)=1$, while $Y=\left\{b_{i}: i=4,5,6,7\right\}$ is a $B$-critical set, $\operatorname{ker}_{B}(G)=\left\{b_{4}, b_{5}, b_{6}\right\}, \operatorname{diadem}_{B}(G)=$ $\left\{b_{i}: i=2, \ldots, 7\right\}$ and $\delta_{0}(B)=2$. 
Theorem 1.3 [11] Let $G=(A, B, E)$. Then the following are true:

(i) the function $\delta$ is supermodular, i.e., $\delta(X \cup Y)+\delta(X \cap Y) \geq \delta(X)+\delta(Y)$ for every $X, Y \subseteq A$ (or $X, Y \subseteq B$ ).

(ii) there is a unique minimal $A$-critical set, namely, $\operatorname{ker}_{A}(G)$, and there is a unique maximal $A$-critical set, namely, $\operatorname{diadem}_{A}(G)$; similarly, for $\operatorname{ker}_{B}(G)$ and $\operatorname{diadem}_{B}(G)$;

(iii) $\mu(G)=|A|-\delta_{0}(A)=|B|-\delta_{0}(B)$.

In this paper we define two new graph parameters, namely, ker and diadem. Further, we analyze their relationships with two other parameters, core and corona, for bipartite graphs.

\section{Preliminaries}

Theorem 2.1 Let $G=(A, B, E)$ be a bipartite graph. Then the following assertions are true:

(i) $d_{c}(G)=\delta_{0}(A)+\delta_{0}(B)$;

(ii) $\alpha(G)=|A|+\delta_{0}(B)=|B|+\delta_{0}(A)=\mu(G)+\delta_{0}(A)+\delta_{0}(B)=\mu(G)+d_{c}(G)$;

(iii) if $X$ is an $A$-critical set and $Y$ is a $B$-critical set, then $X \cup Y$ is a d-critical set;

(iv) if $Z$ is a d-critical independent set, then $Z \cap A$ is an $A$-critical set and $Z \cap B$ is a $B$-critical set;

(v) if $X$ is either an A-critical set or a B-critical set, then there is a matching from $N(X)$ into $X$.

Proof. (i) By Theorems 1.3(iii) and 1.2(ii) we get

$$
\begin{aligned}
d_{c}(G) & =\alpha(G)-\mu(G)=|A|+|B|-2 \mu(G)= \\
& =|A|+|B|-\left(|A|-\delta_{0}(A)\right)-\left(|B|-\delta_{0}(B)\right)=\delta_{0}(A)+\delta_{0}(B) .
\end{aligned}
$$

(ii) Using Theorem 1.3(iii), we infer that

$$
\alpha(G)=|A \cup B|-\mu(G)=|A \cup B|-|A|+\delta_{0}(A)=|B|+\delta_{0}(A) .
$$

Similarly, one can find $\alpha(G)=|A|+\delta_{0}(B)$.

According to part (i), we obtain

$$
\begin{aligned}
\mu(G)+d_{c}(G) & =\mu(G)+\delta_{0}(A)+\delta_{0}(B)= \\
& =|A|-\delta_{0}(A)+\delta_{0}(A)+\delta_{0}(B)=|A|+\delta_{0}(B)=\alpha(G) .
\end{aligned}
$$

(iii) By supermodularity of the function $d$ (Theorem 1.1(iv)) and part (i), we have

$$
\begin{aligned}
d_{c}(G) & \geq d(X \cup Y)=d(X \cup Y)+d(X \cap Y) \geq \\
& \geq d(X)+d(Y)=\delta(X)+\delta(Y)=\delta_{0}(A)+\delta_{0}(B)=d_{c}(G) .
\end{aligned}
$$
then

(iv) Since $Z=(Z \cap A) \cup(Z \cap B)$ and $N(Z \cap A) \cap(Z \cap B)=\emptyset=N(Z \cap B) \cap(Z \cap A)$,

$$
\begin{aligned}
& d(Z \cap A)+d(Z \cap B)=|Z \cap A|-|N(Z \cap A)|+|Z \cap B|-|N(Z \cap B)|= \\
& =|Z \cap A|+|Z \cap B|-|N(Z \cap A)|-|N(Z \cap B)|=|Z|-|N(Z)|=d(Z) .
\end{aligned}
$$


Using the fact that $d(Z)=d_{c}(G)=\delta_{0}(A)+\delta_{0}(B)$, it follows that $d(Z \cap A)=\delta_{0}(A)$ and $d(Z \cap B)=\delta_{0}(B)$.

(v) Let $X$ be an $A$-critical set. Suppose to the contrary that there is no matching from $N(X)$ into $X$. By Hall's Theorem it means that there exists $U \subseteq N(X)$ such that $|N(U) \cap X|<|U|$. Consequently, we obtain

$$
\begin{gathered}
\delta(X-N(U))=|X-N(U)|-|N(X-N(U))|= \\
=|X|-|X \cap N(U)|-(|N(X)|-|U|)= \\
=|X|-|N(X)|+(|U|-|X \cap N(U)|)=\delta_{0}(A)+(|U|-|X \cap N(U)|)>\delta_{0}(A),
\end{gathered}
$$

which contradicts the fact that $X$ is an $A$-critical set.

It is known that a bipartite graph $G$ has a perfect matching if and only if $\alpha(G)=$ $\mu(G)$. Hence using Theorem 2.1(ii), we deduce the following.

Corollary 2.2 [11] A bipartite graph $G=(A, B, E)$ has a perfect matching if and only if $\delta_{0}(A)=0=\delta_{0}(B)$.

Lemma 2.3 Let $G=(A, B, E)$ be a bipartite graph. If $X$ is an $A$-critical set and $Y$ is a B-critical set, then $|X \cap N(Y)|=|N(X) \cap Y|$. Moreover, there is a perfect matching between $X \cap N(Y)$ and $N(X) \cap Y$.

Proof. By Theorem 2.1 $(v)$, there is a matching $M_{1}$ from $N(X)$ into $X$, and a matching $M_{2}$ from $N(Y)$ into $Y$. For each $b \in N(X) \cap Y$, it follows that $M_{1}(b) \in N(b) \subseteq N(Y)$ and $M_{1}(b) \in X$. Hence $M_{1}(b) \in X \cap N(Y)$, which implies $M_{1}(N(X) \cap Y) \subseteq X \cap N(Y)$, and further

$$
|N(X) \cap Y|=\left|M_{1}(N(X) \cap Y)\right| \leq|X \cap N(Y)| .
$$

Similarly, we have

$$
|X \cap N(Y)|=\left|M_{2}(X \cap N(Y))\right| \leq|N(X) \cap Y| .
$$

Consequently, we deduce that $|X \cap N(Y)|=|N(X) \cap Y|$ and the restriction of $M_{1}$ to $N(X) \cap Y$ is a perfect matching from $N(X) \cap Y$ onto $X \cap N(Y)$.

Corollary 2.4 Let $G=(A, B, E)$ is a bipartite graph.

(i) [12] If $X=\operatorname{ker}_{A}(G)$ and $Y$ is a B-critical set, then $X \cap N(Y)=N(X) \cap Y=\emptyset$;

(ii) [11] $\operatorname{ker}_{A}(G) \cap N\left(\operatorname{ker}_{B}(G)\right)=N\left(\operatorname{ker}_{A}(G)\right) \cap \operatorname{ker}_{B}(G)=\emptyset$.

Proof. (i) Assume, to the contrary, that $X \cap N(Y) \neq \emptyset$. By Lemma 2.3, we have $|X \cap N(Y)|=|N(X) \cap Y|$.

If $x \in X-X \cap N(Y)$ has $N(x) \cap Y \neq \emptyset$, then $x \in N(y) \subseteq N(Y)$, which is impossible. Hence $N(X-X \cap N(Y)) \subseteq N(X)-N(X) \cap Y$, and further, we get

$$
\begin{gathered}
|X-X \cap N(Y)|-|N(X-X \cap N(Y))| \geq|X-X \cap N(Y)|-|N(X)-N(X) \cap Y|= \\
=|X|-|X \cap N(Y)|-|N(X)|+|N(X) \cap Y|=\delta(X)=\delta_{0}(A),
\end{gathered}
$$

and this contradicts the minimality of $X$.

(ii) It immediately follows from part (i), when $Y=\operatorname{ker}_{B}(G)$. 


\section{Ker and Core}

Theorem 3.1 Let $X$ be a critical independent set in a graph $G$. Then the following statements are equivalent:

(i) $X=\operatorname{ker}(G)$;

(ii) there is no set $Y \subseteq N(X), Y \neq \emptyset$ such that $|N(Y) \cap X|=|Y|$;

(iii) for each $v \in X$ there exists a matching from $N(X)$ into $X-v$.

Proof. (i) $\Longrightarrow$ (ii) By Theorem 1.1 (ii), there is a matching, say $M$, from $N(\operatorname{ker}(G))$ into $\operatorname{ker}(G)$. Suppose, to the contrary, that there exists some non-empty set $Y \subseteq N(\operatorname{ker}(G))$ such that $|M(Y)|=|N(Y) \cap \operatorname{ker}(G)|=|Y|$. It contradicts the minimality of the set $\operatorname{ker}(G)$, because

$$
d(\operatorname{ker}(G)-N(Y))=d(\operatorname{ker}(G)), \text { while } \operatorname{ker}(G)-N(Y) \varsubsetneqq \operatorname{ker}(G) .
$$

(ii) $\Longrightarrow$ (i) Suppose $X-\operatorname{ker}(G) \neq \emptyset$. By Theorem 1.1 (ii), there is a matching, say $M$, from $N(X)$ into $X$. Since there are no edges connecting vertices from $\operatorname{ker}(G)$ with vertices of $N(X)-N(\operatorname{ker}(G))$, we obtain that $M(N(X)-N(\operatorname{ker}(G))) \subseteq X-\operatorname{ker}(G)$. Moreover, we have that $|N(X)-N(\operatorname{ker}(G))|=|X-\operatorname{ker}(G)|$, otherwise

$$
\begin{aligned}
|X|-|N(X)| & =(|\operatorname{ker}(G)|-|N(\operatorname{ker}(G))|)+(|X-\operatorname{ker}(G)|-|N(X)-N(\operatorname{ker}(G))|)> \\
& >(|\operatorname{ker}(G)|-|N(\operatorname{ker}(G))|)=d_{c}(G) .
\end{aligned}
$$

It means that the set $N(X)-N(\operatorname{ker}(G))$ contradicts the hypothesis of (ii), because

$$
|N(X)-N(\operatorname{ker}(G))|=|X-\operatorname{ker}(G)|=|N(N(X)-N(\operatorname{ker}(G))) \cap X| .
$$

Consequently, the assertion is true.

(ii) $\Longrightarrow$ (iii) By Theorem 1.1(ii), there is a matching, say $M$, from $N(X)$ into $X$. Suppose, to the contrary, that there is no matching from $N(X)$ into $X-v$. By Hall's Theorem, it implies the existence of a set $Y \subseteq N(X)$ such that $|N(Y) \cap X|=|Y|$, which contradicts the hypothesis of (ii).

(iii) $\Longrightarrow$ (ii) Suppose, to the contrary, there is a non-empty subset $Y$ of $N(X)$ such that $|N(Y) \cap X|=|Y|$. Let $v \in N(Y) \cap X$. Hence, we get $|N(Y) \cap X-v|<|Y|$. Then, by Hall's Theorem, it is impossible to find a matching from $N(X)$ into $X-v$, which contradicts the hypothesis of (iii).

Lemma 3.2 If $G=(A, B, E)$ is a bipartite graph with a perfect matching, say $M$, $S \in \Omega(G), X \in \operatorname{Ind}(G), X \subseteq V(G)-S$, and $G[X \cup M(X)]$ is connected, then

$$
X^{1}=X \cup M((N(X) \cap S)-M(X))
$$

is an independent set, and $G\left[X^{1} \cup M\left(X^{1}\right)\right]$ is connected.

Proof. Let us show that the set $M((N(X) \cap S)-M(X))$ is independent. Suppose, to the contrary, that there exist $v_{1}, v_{2} \in M((N(X) \cap S)-M(X))$ such that $v_{1} v_{2} \in E(G)$. Hence $M\left(v_{1}\right), M\left(v_{2}\right) \in(N(X) \cap S)-M(X)$. 
If $M\left(v_{1}\right)$ and $M\left(v_{2}\right)$ have a common neighbor $w \in X$, then $\left\{v_{1}, v_{2}, M\left(v_{2}\right), w, M\left(v_{1}\right)\right\}$ spans $C_{5}$, which is forbidden for bipartite graphs.

Otherwise, let $w_{1}, w_{2} \in X$ be neighbors of $M\left(v_{1}\right)$ and $M\left(v_{2}\right)$, respectively. Since $G[X \cup M(X)]$ is connected, there is a path with even number of edges connecting $w_{1}$ and $w_{2}$. Together with $\left\{w_{1}, M\left(v_{1}\right), v_{1}, v_{2}, M\left(v_{2}\right), w_{2}\right\}$ this path produces a cycle of odd length in contradiction with the hypothesis on $G$ being a bipartite graph.

To complete the proof of independence of the set

$$
X^{1}=X \cup M((N(X) \cap S)-M(X))
$$

it is enough to demonstrate that there are no edges connecting vertices of $X$ and $M((N(X) \cap S)-M(X))$.

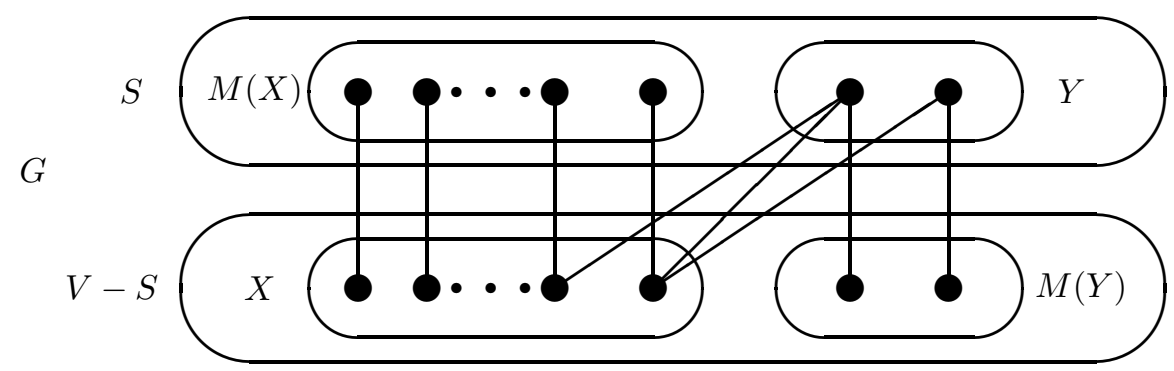

Figure 3: $S \in \Omega(G), Y=(N(X) \cap S)-M(X)$ and $X^{1}=X \cup M(Y)$.

Assume, to the contrary, that there is $v w \in E$, such that $v \in M((N(X) \cap S)-M(X))$ and $w \in X$. Since $M(v) \in(N(X) \cap S)-M(X)$ and $G[X \cup M(X)]$ is connected, it follows that there exists a path with an odd number of edges connecting $M(v)$ to $w$. This path together with the edges $v w$ and $v M(v)$ produces cycle of odd length, in contradiction with the bipartiteness of $G$.

Finally, since $G[X \cup M(X)]$ is connected, $G\left[X^{1} \cup M\left(X^{1}\right)\right]$ is connected as well, by definitions of set functions $N$ and $M$.

Theorem 1.1(vii) claims that $\operatorname{ker}(G) \subseteq \operatorname{core}(G)$ for every graph.

Theorem 3.3 If $G$ is a bipartite graph, then $\operatorname{ker}(G)=\operatorname{core}(G)$.

Proof. The assertions are clearly true, whenever $\operatorname{core}(G)=\emptyset$, i.e., for $G$ having a perfect matching. Assume that $\operatorname{core}(G) \neq \emptyset$.

Let $S \in \Omega(G)$ and $M$ be a maximum matching. By Theorem $1.2(i), M$ matches $V(G)-S$ into $S$, and $N(\operatorname{core}(G))$ into core $(G)$.

According to Theorem 3.1 it is sufficient to show that there is no set $Z \subseteq N(\operatorname{core}(G))$, $Z \neq \emptyset$, such that $|N(Z) \cap \operatorname{core}(G)|=|Z|$.

Suppose, to the contrary, that there exists a non-empty set $Z \subseteq N(\operatorname{core}(G))$ such that $|N(Z) \cap \operatorname{core}(G)|=|Z|$. Let $Z_{0}$ be a minimal non-empty subset of $N(\operatorname{core}(G))$ enjoying this equality.

Clearly, $H=G\left[Z_{0} \cup M\left(Z_{0}\right)\right]$ is bipartite, because it is a subgraph of a bipartite graph. Moreover, the restriction of $M$ on $H$ is a perfect matching. 
Claim 1. $Z_{0}$ is independent.

Since $H$ is a bipartite graph with a perfect matching it has two maximum independent sets at least. Hence there exists $W \in \Omega(H)$ different from $M\left(Z_{0}\right)$. Thus $W \cap Z_{0} \neq \emptyset$. Therefore, $N\left(W \cap Z_{0}\right) \cap \operatorname{core}(G)=M\left(W \cap Z_{0}\right)$. Consequently,

$$
\left|N\left(W \cap Z_{0}\right) \cap \operatorname{core}(G)\right|=\left|M\left(W \cap Z_{0}\right)\right|=\left|W \cap Z_{0}\right| .
$$

Finally, $W \cap Z_{0}=Z_{0}$, because $Z_{0}$ has been chosen as a minimal subset of $N(\operatorname{core}(G))$ such that $\left|N\left(Z_{0}\right) \cap \operatorname{core}(G)\right|=\left|Z_{0}\right|$. Since $\left|Z_{0}\right|=\alpha(H)=|W|$ we conclude with $W=Z_{0}$, which means, in particular, that $Z_{0}$ is independent.

Claim 2. $H$ is a connected graph.

Otherwise, for any connected component of $H$, say $\tilde{H}$, the set $V(\tilde{H}) \cap Z_{0}$ contradicts the minimality property of $Z_{0}$.

Claim 3. $Z_{0} \cup\left(\operatorname{core}(G)-M\left(Z_{0}\right)\right)$ is independent.

By Claim $1 Z_{0}$ is independent. The equality $\left|N\left(Z_{0}\right) \cap \operatorname{core}(G)\right|=\left|Z_{0}\right|$ implies $N\left(Z_{0}\right) \cap \operatorname{core}(G)=M\left(Z_{0}\right)$, which means that there are no edges connecting $Z_{0}$ and core $(G)-M\left(Z_{0}\right)$. Consequently, $Z_{0} \cup\left(\operatorname{core}(G)-M\left(Z_{0}\right)\right)$ is independent.

Claim 4. $Z_{0} \cup\left(\operatorname{core}(G)-M\left(Z_{0}\right)\right)$ is included in a maximum independent set.

Let $Z_{i}=M\left(\left(N\left(Z_{i-1}\right) \cap S\right)-M\left(Z_{i-1}\right)\right), 1 \leq i<\infty$. By Lemma 3.2 all the sets $Z^{i}=\bigcup_{0 \leq j \leq i} Z_{j}, 1 \leq i<\infty$ are independent. Define

$$
Z^{\infty}=\bigcup_{0 \leq i \leq \infty} Z_{i}
$$

which is, actually, the largest set in the sequence $\left\{Z^{i}, 1 \leq i<\infty\right\}$.

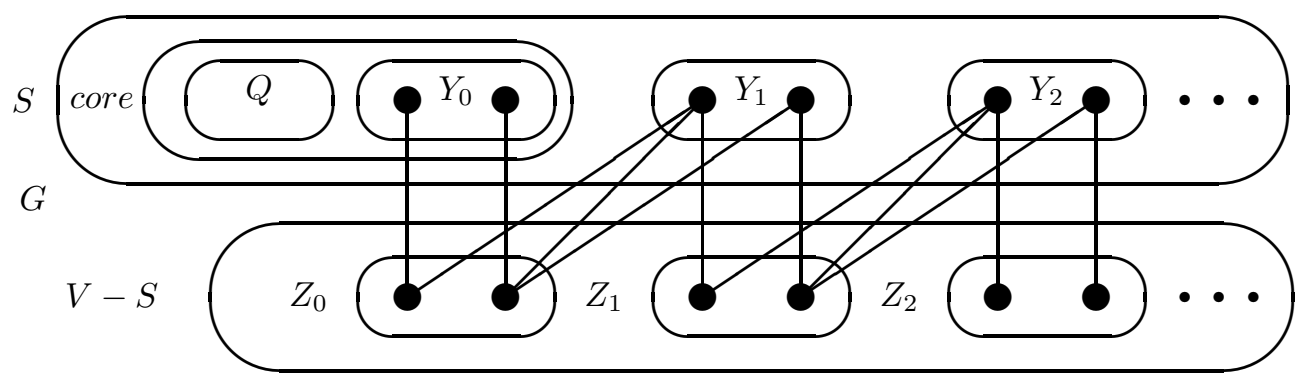

Figure 4: $S \in \Omega(G), Q=$ core $(G)-M\left(Z_{0}\right), Y_{0}=M\left(Z_{0}\right), Y_{1}=\left(N\left(Z_{0}\right)-M\left(Z_{0}\right)\right) \cap S$, $Y_{2}=\ldots$ and $Z_{i}=M\left(Y_{i}\right), i=1,2, \ldots$.

The inclusion

$$
Z_{0} \cup\left(\operatorname{core}(G)-M\left(Z_{0}\right)\right) \subseteq\left(S-M\left(Z^{\infty}\right)\right) \cup Z^{\infty}
$$

is justified by the definition of $Z^{\infty}$. 
Since $\left|M\left(Z^{\infty}\right)\right|=\left|Z^{\infty}\right|$ we obtain $\left|\left(S-M\left(Z^{\infty}\right)\right) \cup Z^{\infty}\right|=|S|$. According to the definition of $Z^{\infty}$ the set

$$
\left(N\left(Z^{\infty}\right) \cap S\right)-M\left(Z^{\infty}\right)
$$

is empty. In other words, the set $\left(S-M\left(Z^{\infty}\right)\right) \cup Z^{\infty}$ is independent. Therefore, we arrive at

$$
\left(S-M\left(Z^{\infty}\right)\right) \cup Z^{\infty} \in \Omega(G) .
$$

Consequently, $\left(S-M\left(Z^{\infty}\right)\right) \cup Z^{\infty}$ is a desired enlargement of $Z_{0} \cup\left(\operatorname{core}(G)-M\left(Z_{0}\right)\right)$. Claim 5. $\operatorname{core}(G) \cap\left(\left(S-M\left(Z^{\infty}\right)\right) \cup Z^{\infty}\right)=\operatorname{core}(G)-M\left(Z_{0}\right)$.

The only part of $\left(S-M\left(Z^{\infty}\right)\right) \cup Z^{\infty}$ that interacts with core $(G)$ is the subset

$$
Z_{0} \cup\left(\operatorname{core}(G)-M\left(Z_{0}\right)\right)
$$

Hence we obtain

$$
\begin{gathered}
\operatorname{core}(G) \cap\left(\left(S-M\left(Z^{\infty}\right)\right) \cup Z^{\infty}\right)= \\
=\operatorname{core}(G) \cap\left(Z_{0} \cup\left(\operatorname{core}(G)-M\left(Z_{0}\right)\right)\right)=\operatorname{core}(G)-M\left(Z_{0}\right) .
\end{gathered}
$$

Since $Z_{0}$ is non-empty, by Claim 5 we arrive at the following contradiction

$$
\operatorname{core}(G) \nsubseteq\left(S-M\left(Z^{\infty}\right)\right) \cup Z^{\infty} \in \Omega(G) \text {. }
$$

Finally, we conclude with the fact there is no set $Z \subseteq N(\operatorname{core}(G)), Z \neq \emptyset$ such that $|N(Z) \cap \operatorname{core}(G)|=|Z|$, which, by Theorem 3.1, means that $\operatorname{core}(G)$ and $\operatorname{ker}(G)$ coincide.

Notice that there are non-bipartite graphs enjoying the equality $\operatorname{ker}(G)=\operatorname{core}(G)$; e.g., the graphs from Figure 5. Notice that only $G_{1}$ is a König-Egerváry graph.
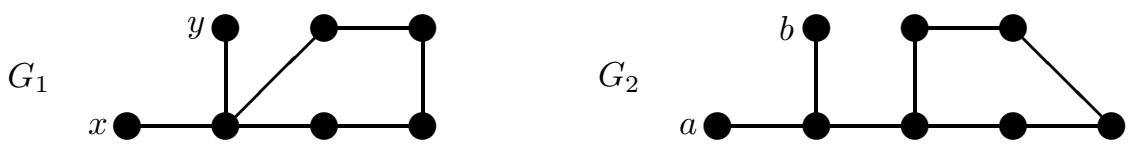

Figure 5: $\operatorname{core}\left(G_{1}\right)=\operatorname{ker}\left(G_{1}\right)=\{x, y\}$ and $\operatorname{core}\left(G_{2}\right)=\operatorname{ker}\left(G_{2}\right)=\{a, b\}$.

There is a non-bipartite König-Egerváry graph $G$, such that $\operatorname{ker}(G) \neq \operatorname{core}(G)$. For instance, the graph $G_{1}$ from Figure 6 has $\operatorname{ker}\left(G_{1}\right)=\{x, y\}$, while $\operatorname{core}\left(G_{1}\right)=\{x, y, u, v\}$. The graph $G_{2}$ from Figure 6 has $\operatorname{ker}\left(G_{2}\right)=\emptyset$, while core $\left(G_{2}\right)=\{w\}$.
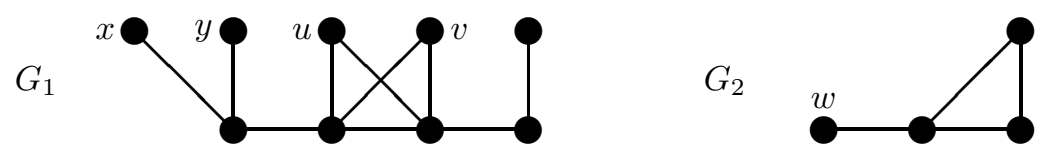

Figure 6: Both $G_{1}$ and $G_{2}$ are König-Egerváry graphs. Only $G_{2}$ has a perfect matching. 


\section{Ker and Diadem}

Proposition 4.1 [7] If $G$ is a König-Egerváry graph, then

$N(\operatorname{core}(G))=\cap\{V(G)-S: S \in(G)\}$, i.e., $N(\operatorname{core}(G))=V(G)-\operatorname{corona}(G)$.

There is a non-König-Egerváry graph $G$ with $V(G)=N(\operatorname{core}(G)) \cup \operatorname{corona}(G)$; e.g., the graph $G$ from Figure 7 .

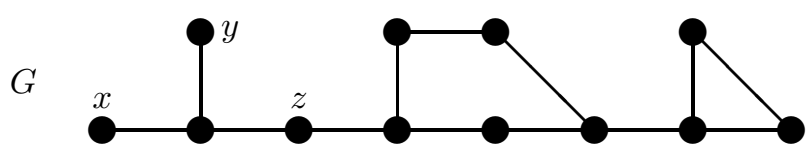

Figure 7: $G$ is not a König-Egerváry graph, and core $(G)=\{x, y, z\}$.

Theorem 4.2 If $G$ is a König-Egerváry graph, then

(i) $|\operatorname{corona}(G)|+|\operatorname{core}(G)|=2 \alpha(G)$;

(ii) $\operatorname{diadem}(G)=\operatorname{corona}(G)$, while $\operatorname{diadem}(G) \subseteq \operatorname{corona}(G)$ is true for every graph;

(iii) $|\operatorname{ker}(G)|+|\operatorname{diadem}(G)| \leq 2 \alpha(G)$.

Proof. (i) Using Theorem 1.2(ii) and Proposition 4.1 we infer that

$$
\begin{gathered}
|\operatorname{corona}(G)|+|\operatorname{core}(G)|=|\operatorname{corona}(G)|+|N(\operatorname{core}(G))|+|\operatorname{core}(G)|-|N(\operatorname{core}(G))|= \\
=|V(G)|+d_{c}(G)=\alpha(G)+\mu(G)+d_{c}(G)=2 \alpha(G) .
\end{gathered}
$$

as claimed.

(ii) Every $S \in \Omega(G)$ is $d$-critical, by Theorem 1.2(ii). Further, Theorem [1.2(ii) ensures that corona $(G) \subseteq \operatorname{diadem}(G)$. On the other hand, each critical independent set is included in a maximum independent set, according to Theorem 1.1(iii). Thus, we have $\operatorname{diadem}(G) \subseteq \operatorname{corona}(G)$. Consequently, the equality $\operatorname{diadem}(G)=$ corona $(G)$ holds.

(iii) It follows by combining parts (i),(ii) and Theorem 1.1)(vii).

Notice that the graph from Figure 7 has $|\operatorname{corona}(G)|+|\operatorname{core}(G)|=13>12=2 \alpha(G)$.

For a König-Egerváry graph with $|\operatorname{ker}(G)|+|\operatorname{diadem}(G)|<2 \alpha(G)$ see Figure 6. Figure 7 shows that it is possible for a graph to have $\operatorname{diadem}(G) \varsubsetneqq \operatorname{corona}(G)$ and $\operatorname{ker}(G) \varsubsetneqq \operatorname{core}(G)$.

$G_{1}$

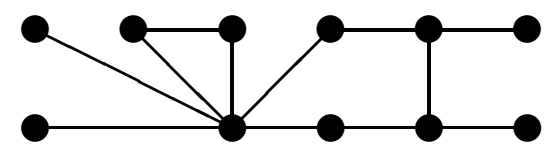

$G_{2}$

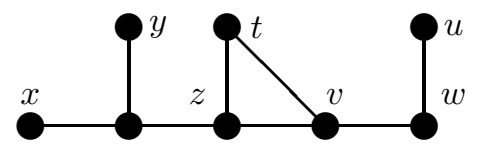

Figure 8: $G_{1}$ is a non-bipartite König-Egerváry graph, such that $\operatorname{ker}\left(G_{1}\right)=\operatorname{core}\left(G_{1}\right)$ and $\operatorname{diadem}\left(G_{1}\right)=\operatorname{corona}\left(G_{1}\right) ; G_{2}$ is a non-König-Egerváry graph, such that $\operatorname{ker}(G)=$ $\operatorname{core}(G)=\{x, y\} ; \operatorname{diadem}(G) \cup\{z, t, v, w\}=\operatorname{corona}(G)$.

The combination of $\operatorname{diadem}(G) \varsubsetneqq \operatorname{corona}(G)$ and $\operatorname{ker}(G)=\operatorname{core}(G)$ is realized in Figure 8 .

Now we are ready to describe both ker and diadem of a bipartite graph in terms of its bipartition. 
Theorem 4.3 Let $G=(A, B, E)$ be a bipartite graph. Then the following assertions are true:

(i) $\operatorname{ker}_{A}(G) \cup \operatorname{ker}_{B}(G)=\operatorname{ker}(G)$;

(ii) $|\operatorname{ker}(G)|+|\operatorname{diadem}(G)|=2 \alpha(G)$;

(iii) $\left|\operatorname{ker}_{A}(G)\right|+\left|\operatorname{diadem}_{B}(G)\right|=\left|\operatorname{ker}_{B}(G)\right|+\left|\operatorname{diadem}_{A}(G)\right|=\alpha(G)$;

(iv) $\operatorname{diadem}_{A}(G) \cup \operatorname{diadem}_{B}(G)=\operatorname{diadem}(G)$.

Proof. (i) By Theorem 2.1(iii), $\operatorname{ker}_{A}(G) \cup \operatorname{ker}_{B}(G)$ is $d$-critical in $G$. Moreover, $\operatorname{ker}_{A}(G) \cup \operatorname{ker}_{B}(G)$ is independent in accordance with Corollary 2.4 Assume that $\operatorname{ker}_{A}(G) \cup \operatorname{ker}_{B}(G)$ is not minimal. Hence the unique minimal $d$-critical set of $G$, say $Z$, is a proper subset of $\operatorname{ker}_{A}(G) \cup \operatorname{ker}_{B}(G)$, by Theorem 1.1)(vi). According to Theorem

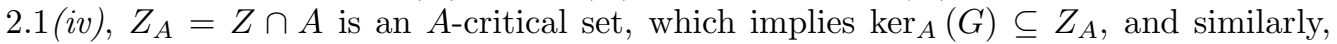
$\operatorname{ker}_{B}(G) \subseteq Z_{B}$. Consequently, we get that $\operatorname{ker}_{A}(G) \cup \operatorname{ker}_{B}(G) \subseteq Z$, in contradiction with the fact that $\operatorname{ker}_{A}(G) \cup \operatorname{ker}_{B}(G) \neq Z \subset \operatorname{ker}_{A}(G) \cup \operatorname{ker}_{B}(G)$.

(ii), (iii), (iv) By Corollary [2.4, we have

$$
\left|\operatorname{ker}_{A}(G)\right|-\delta_{0}(A)+\left|\operatorname{diadem}_{B}(G)\right|=\left|N\left(\operatorname{ker}_{A}(G)\right)\right|+\left|\operatorname{diadem}_{B}(G)\right| \leq|B| .
$$

Hence, according to Theorem 2.1](ii), it follows that

$$
\left|\operatorname{ker}_{A}(G)\right|+\left|\operatorname{diadem}_{B}(G)\right| \leq|B|+\delta_{0}(A)=\alpha(G) .
$$

Changing the roles of $A$ and $B$, we obtain

$$
\left|\operatorname{ker}_{B}(G)\right|+\left|\operatorname{diadem}_{A}(G)\right| \leq \alpha(G) .
$$

By Theorem 2.1 (iv), $\operatorname{diadem}(G) \cap A$ is $A$-critical and diadem $(G) \cap B$ is $B$-critical. Hence $\operatorname{diadem}(G) \cap A \subseteq \operatorname{diadem}_{A}(G)$ and $\operatorname{diadem}(G) \cap B \subseteq \operatorname{diadem}_{B}(G)$. It implies both the inclusion diadem $(G) \subseteq \operatorname{diadem}_{A}(G) \cup \operatorname{diadem}_{B}(G)$, and the inequality

$$
|\operatorname{diadem}(G)| \leq\left|\operatorname{diadem}_{A}(G)\right|+\left|\operatorname{diadem}_{B}(G)\right| .
$$

Combining Theorem 3.3. Theorem 4.2(ii) and part (i) with the above inequalities, we deduce

$$
\begin{gathered}
2 \alpha(G) \geq\left|\operatorname{ker}_{A}(G)\right|+\left|\operatorname{ker}_{B}(G)\right|+\left|\operatorname{diadem}_{A}(G)\right|+\left|\operatorname{diadem}_{B}(G)\right| \geq \\
=|\operatorname{ker}(G)|+|\operatorname{diadem}(G)|=|\operatorname{core}(G)|+|\operatorname{corona}(G)|=2 \alpha(G) .
\end{gathered}
$$

Consequently, we infer that

$$
\begin{gathered}
\left|\operatorname{diadem}_{A}(G)\right|+\left|\operatorname{diadem}_{B}(G)\right|=|\operatorname{diadem}(G)|, \\
|\operatorname{ker}(G)|+\mid \operatorname{diadem}_{(G)}(=2 \alpha(G), \\
\left|\operatorname{ker}_{A}(G)\right|+\left|\operatorname{diadem}_{B}(G)\right|=\left|\operatorname{ker}_{B}(G)\right|+\left|\operatorname{diadem}_{A}(G)\right|=\alpha(G) .
\end{gathered}
$$

Since diadem $(G) \subseteq \operatorname{diadem}_{A}(G) \cup \operatorname{diadem}_{B}(G)$ and $\operatorname{diadem}_{A}(G) \cap \operatorname{diadem}_{B}(G)=\emptyset$, we finally obtain that

$$
\operatorname{diadem}_{A}(G) \cup \operatorname{diadem}_{B}(G)=\operatorname{diadem}(G),
$$

as claimed. 


\section{Conclusions}

In this paper we focus on interconnections between ker, core, diadem, and corona for König-Egerváry graphs, in general, and bipartite graphs, in particular.

In [9] we showed that $2 \alpha(G) \leq|\operatorname{core}(G)|+|\operatorname{corona}(G)|$ is true for every graph. By Theorem $4.2(i)$, this equality is true whenever $G$ is a König-Egerváry graph.

According to Theorem 1.1)(vii), $\operatorname{ker}(G) \subseteq \operatorname{core}(G)$ for every graph. On the other hand, Theorem 1.1(iii) implies the inclusion $\operatorname{diadem}(G) \subseteq \operatorname{corona}(G)$. Hence

$$
|\operatorname{ker}(G)|+|\operatorname{diadem}(G)| \leq|\operatorname{core}(G)|+|\operatorname{corona}(G)|
$$

for each graph $G$. These remarks together with Theorem 4.2(iii) motivate the following.

Conjecture 5.1 $|\operatorname{ker}(G)|+|\operatorname{diadem}(G)| \leq 2 \alpha(G)$ is true for every graph $G$.

When it is proved one can conclude that the following inequalities:

$$
|\operatorname{ker}(G)|+|\operatorname{diadem}(G)| \leq 2 \alpha(G) \leq|\operatorname{core}(G)|+|\operatorname{corona}(G)|
$$

hold for every graph $G$.

\section{References}

[1] S. Butenko, S. Trukhanov, Using critical sets to solve the maximum independent set problem, Operations Research Letters 35 (2007) 519-524.

[2] R. W. Deming, Independence numbers of graphs - an extension of the KönigEgerváry theorem, Discrete Mathematics 27 (1979) 23-33.

[3] E. Egerváry, On combinatorial properties of matrices, Matematikai Lapok 38 (1931) $16-28$.

[4] D. König, Graphen und matrizen, Matematikai Lapok 38 (1931) 116-119.

[5] C. E. Larson, A note on critical independence reductions, Bulletin of the Institute of Combinatorics and its Applications 5 (2007) 34-46.

[6] V. E. Levit, E. Mandrescu, Combinatorial properties of the family of maximum stable sets of a graph, Discrete Applied Mathematics 117 (2002) 149-161.

[7] V. E. Levit, E. Mandrescu, On $\alpha^{+}$-stable König-Egerváry graphs, Discrete Mathematics 263 (2003) 179-190.

[8] V. E. Levit, E. Mandrescu, Critical independent sets and König-Egerváry graphs, Graphs and Combinatorics (2011) (accepted), arXiv:0906.4609v2 [math.CO], 8 pp.

[9] V. E. Levit, E. Mandrescu, A set and collection lemma, (2011) arXiv:1101.4564v1 [cs.DM], 6 pp. 
[10] V. E. Levit, E. Mandrescu, Vertices belonging to all critical independent sets of a graph, (2011) arXiv:1102.0401v1 [cs.DM], 9 pp.

[11] O. Ore, Graphs and matching theorems, Duke Mathematical Journal 22 (1955) 625639.

[12] O. Ore, Theory of Graphs, AMS Colloquium Publications 38 (1962) AMS.

[13] F. Sterboul, A characterization of the graphs in which the transversal number equals the matching number, Journal of Combinatorial Theory Series B 27 (1979) 228-229.

[14] C. Q. Zhang, Finding critical independent sets and critical vertex subsets are polynomial problems, SIAM Journal of Discrete Mathematics 3 (1990) 431-438. 